\title{
AED for Paediatric Use, Implications in the Design of Shock Advice Algorithms
}

\author{
Sofia Ruiz de Gauna, Jesus Ruiz, Unai Irusta and Elisabete Aramendi \\ University of the Basque Country \\ Spain
}

\section{Introduction}

Sudden cardiac death (SCD) is the single most important cause of death in the adult population of the industrialised world (Jacobs et al., 2004). It is estimated that SCD accounts for an average of 100 to 200 deaths per 100000 adults over 35 years and represents $50 \%$ of all heart-related death (Myerburg, 2001). Most sudden cardiac arrests occur out of hospital, and the annual incidence of out of hospital cardiac arrest (OHCA) treated by emergency medical services in the US is 55 per 100000 population (Myerburg, 2001).

Ventricular fibrillation (VF) is the initial rhythm in $40 \%$ of cardiac arrest cases (Rea et al., 2004). Survival rates in untreated VF cardiac arrest decrease by 7 to $10 \%$ per minute (Larsen et al., 1993; Valenzuela et al., 1997), as the heart function deteriorates. Although many victims present VF or ventricular tachycardia (VT) at the time of collapse, by the time the first ECG is recorded the rhythm has deteriorated to asystole (Waalewijn et al., 2002). The estimated survival rate in all cases of OHCA is a poor $8.4 \%$, but rises to $17.7 \%$ when the victim presents VF as initial rhythm (Rea et al., 2004). Consequently, early intervention is critical for the survival of OHCA victims.

In the early 1990s, the American Heart Association (AHA) established the chain of survival metaphor to describe the sequence of actions to treat OHCA. The chain of survival consists of four links: early access, early cardiopulmonary resuscitation, early defibrillation and early advanced cardiac life support. Defibrillation is normally administered using an automated external defibrillator (AED). The AED analyses the rhythm acquired through two electrode pads and delivers an electrical shock if a lethal ventricular arrhythmia - VF or rapid VT - is detected. The shock advice algorithm (SAA) of an AED analyses the ECG to discriminate shockable from non-shockable rhythms. Given a database of classified ECG records, the performance of the SAA is evaluated in terms of the proportion of correctly identified shockable - sensitivity and non-shockable - specificity - rhythms, which must exceed the minimum values set by the AHA (Kerber et al., 1997).

SCD is 10 times less frequent in children than in adults. However, only in the US, an estimated 16000 children die each year from sudden cardiac arrest (Sirbaugh et al., 1999). Moreover, the social and emotional impact of the death of a child is enormous because of the greater life expectancy. Paediatric cardiac arrest constitutes less than $10 \%$ of all OHCA, and cardiac arrest due to arrhythmias is also less frequent in children than in adults. VF is the initially recorded 
rhythm in $3.8-19 \%$ of paediatric OHCA cases, and more importantly, VF is the arrhythmia associated with the highest survival rate (Biarent et al., 2005).

As late as 2000, no conclusive study existed on the use of AED in children under 8 years of age. By 2003 the International Liaison Committee on Resuscitation (ILCOR) recommended the use of AED in children 1-8 years of age (Samson et al., 2003), based on the evidence provided in two independent studies (Atkinson et al., 2003; Cecchin et al., 2001). Since 2005 the Resuscitation guidelines ${ }^{1}$ reflect the need of adapting AED for paediatric use. This involves adjusting the defibrillation pads and the defibrillation energy dose, but also, demonstrating that the SAA are accurate in the detection of paediatric arrhythmias. In fact, the first studies on the use of AED in children showed that two adult SAA from commercial AED accurately identified many paediatric rhythms (Atkinson et al., 2003; Cecchin et al., 2001). The specificity for non-shockable rhythms and the sensitivity for VF were above the values recommended by the AHA. However, those studies failed to meet AHA criteria for shockable paediatric VT. In 2008, a third study showed that a SAA designed for adult patients did not meet AHA criteria for non-shockable paediatric supraventricular tachycardia (SVT) (Atkins et al., 2008).

SAA are based on the combination of several discrimination parameters computed from the surface ECG. There are differences in rate, amplitude and ECG wave morphology between paediatric and adult rhythms (Aramendi et al., 2010; Cecchin et al., 2001; Rustwick et al., 2007). The faster heart rates and shorter QRS durations of paediatric rhythms produce differences in the values of the discrimination parameters which may affect the performance of SAA designed for adult use. Some of these differences have been previously assessed (Aramendi et al., 2010; Irusta et al., 2008; Ruiz de Gauna et al., 2008). However, based on the thorough analysis presented in this chapter, adequate strategies can be defined to either adapt (Atkins et al., 2008; Irusta \& Ruiz, 2009) or redesign adult SAA algorithms to be valid also for paediatric use.

The discrimination parameters analysed in this study quantify features of the ECG signal in three domains: slope, frequency and time. The parameters are computed for a database of more than 1900 ECG records from adult and paediatric patients. Section 2 describes the database in terms of numbers of rhythms and patients, as well as the ECG record sources and the rhythm classification process. Then, in section 3 paediatric and adult heart rates are compared for the different age groups. The differences in the values of the discrimination parameters between adult and paediatric rhythms are assessed in section 4 . The differences are quantified in terms of the statistical distributions of the values of the parameters, both for shockable and non-shockable rhythms. Emphasis is made on the influence of the age group on the values of the parameters. Then, the discriminative power of each parameter is assessed through the Receiver Operating Characteristic (ROC) curve and the sensitivity/specificity values. The analysis reveals important aspects that must be addressed when a SAA is adapted or designed for paediatric use. Finally, section 5 discusses strategies to design reliable SAA for adults and children and puts forward the main conclusions extracted from the study.

\section{Collecting ECG recordings from adult and paediatric patients}

The framework for the assessment of AED SAA was established by an AHA statement in 1997 (Kerber et al., 1997). The statement describes the composition of the databases used to

\footnotetext{
${ }^{1}$ The latest version of the guidelines was released in 2010 (Biarent et al., 2010).
} 
develop and test SAA, including the types of rhythms and the minimum number of records per rhythm type. It also defines the minimum sensitivities and specificities of SAA for those rhythm types, these values are compiled in table 1 . Furthermore, the statement mentions that although a database can contain several records from the same patient there can only be one record of each rhythm type per patient.

Currently there exist no public database of ECG records compliant with the AHA statement. Each AED manufacturer compiles its own data, which must include paediatric rhythms if the AED will treat children. However, the studies describing paediatric databases (Atkins et al., 2008; Atkinson et al., 2003; Cecchin et al., 2001) report less shockable rhythms than those specified by the AHA because paediatric ventricular arrhythmias are scarce.

In this study we use a database composed of adult and paediatric ECG records collected from in- and out-of-hospital sources. Three cardiologists assigned a rhythm type and a shock/no-shock recommendation to each record. For potentially shockable rhythms, the criteria to determine the shock/no-shock recommendation were: the patient is unresponsive, has no palpable pulse, and an unknown age (Cecchin et al., 2001). Diagnostic discrepancies among the reviewers were further discussed, and a consensus decision for the shock/no-shock diagnosis was agreed after the assessment of the risks of each potential recommendation.

The database contains shockable rhythms (VF and rapid VT) and the most representative non-shockable rhythms: Normal Sinus Rhythm (NSR) and SVT. SVT were treated separately because adult SAA have been shown to misdiagnose high rate paediatric SVT (Atkins et al., 2008). Furthermore, VT should only be shocked when its rate exceeds a threshold. In fact, the AHA statement allows the manufacturer to specify this threshold because tolerance to VT varies widely among patients. We considered VT shockable for rates above $150 \mathrm{bpm}$ in adults and $20 \mathrm{bpm}$ above age-matched normal rate in children (Atkinson et al., 2003). Table 1 is a summary of the database, where the paediatric data is divided in three age groups: under 1

\begin{tabular}{|c|c|c|c|c|c|c|}
\hline \multirow[b]{2}{*}{ Rhythms } & \multirow[b]{2}{*}{ Adult } & \multicolumn{3}{|c|}{ Paediatric } & \multicolumn{2}{|c|}{ AHA goal } \\
\hline & & $<1 y$ & $1 y-8 y$ & $>8 y$ & Records & Sens/Esp \\
\hline \multicolumn{7}{|l|}{ Shockable } \\
\hline Coarse VF ${ }^{\mathrm{a}}$ & $374(374)$ & $3(1)$ & $18(11)$ & $37(10)$ & 200 & $90 \%$ \\
\hline Rapid VT & $200(200)$ & $8(4)$ & 39 (19) & $19(13)$ & 50 & $75 \%$ \\
\hline \multicolumn{7}{|l|}{ Non-shockable } \\
\hline NSR & $292(292)$ & $14(13)$ & $312(280)$ & $214(161)$ & 100 & $99 \%$ \\
\hline SVT & 89 (89) & $38(29)$ & 147 (103) & 137 (104) & 30 & $95 \%$ \\
\hline Total & $955(820)$ & $63(39)$ & $516(357)$ & $407(216)$ & - & - \\
\hline
\end{tabular}

a Peak-to-peak amplitude above $200 \mu \mathrm{V}$.

Table 1. Number of records per rhythm class in the adult and paediatric databases, the number of patients is indicated in parenthesis. The AHA statement sets the minimum figures per rhythm class, both in terms of number of records to test SAA and sensitivity/specificity scores of the SAA. 
year (infants), 1-8 years of age (ILCOR recommendation) and above 8 years. All records were stored with a common format, and a sampling frequency of $f_{s}=250 \mathrm{~Hz}$.

The following sections describe the origin and the characteristics of the adult and paediatric records.

\subsection{ECG records from adult patients}

The adult database contains 955 records from 820 patients, 574 non-shockable records from 541 patients and 381 shockable records from 351 patients. The database is fully compliant with the AHA statement for the rhythm categories used in this study. The mean duration of the records was $13.0 \pm 5.3 \mathrm{~s}, 15.4 \pm 4.2 \mathrm{~s}$ for the non-shockable and $11.4 \pm 5.4 \mathrm{~s}$ for the shockable records.

The adult records were obtained from three sources. 251 non-shockable and 63 shockable records were extracted from Public ECG databases ${ }^{2}$. The adult data also includes 127 shockable and 325 non-shockable records from in-hospital electrophysiology (EP) studies and intensive care units obtained at two Spanish hospitals (Basurto and Donostia hospitals). Finally, the database contains 3 non-shockable and 186 shockable out-of-hospital records from the Spanish emergency services in Madrid and the Basque Country.

Public databases are available in digital format with different sampling rates and storage formats. In-hospital data was gathered in digital format (Prucka Cardiolab and EP-Tracer systems) or as printed ECG paper strips. All the out-of-hospital data came in paper format from AED printouts.

\subsection{ECG records from paediatric patients}

The paediatric database contains 986 records from 612 paediatric and adolescent patients aged between 1 day and 20 years (mean age $7.1 \pm 4.5$ years). There are 862 non-shockable records from 579 patients and 124 shockable records from 49 patients. The mean duration of the records was $13.7 \pm 9.0 \mathrm{~s}, 14.1 \pm 9.3 \mathrm{~s}$ for the non-shockable and $10.9 \pm 4.9 \mathrm{~s}$ for the shockable records.

Although the non-shockable portion of the database is compliant with the AHA statement, the shockable portion is not. There are only $58 \mathrm{VF}$ from 22 patients and $66 \mathrm{VT}$ from 36 patients, well below the 250 fatal ventricular arrhythmias (200 VF and 50 VT) specified in the AHA statement. Furthermore in the paediatric database rhythm repetition - one patient contributing more than one record within a rhythm type - was allowed when the morphology of the rhythms was sufficiently different. A low number of ventricular arrhythmias and rhythm repetition ease the terms imposed by the AHA statement, however all studies on the use of AED in children have followed this path due to the scarceness of paediatric ventricular arrhythmias (Atkins et al., 2008; Atkinson et al., 2003; Cecchin et al., 2001).

All the paediatric records were collected in-hospital, from archived paper and digital EP studies (Prucka Cardiolab and EP-Tracer systems). The records were retrospectively obtained in five Spanish hospitals: Cruces, Donostia, La Paz , Gregorio Marañón and San Joan de Deu. The accurate discrimination of SVT and VT based on a single lead ECG is hard in the paediatric case (Irusta \& Ruiz, 2009). In fact, there were a number of paediatric SVT and VT cases were the consensus decision between the cardiologists was not possible, Fig. 1 shows four

${ }^{2}$ The MIT-BIH arrhythmia, the AHA and the Creighton University Ventricular Tachyarrhythmia databases. 
examples. Discrepancies were resolved by adopting the original interpretation from the physician aware of the clinical history of the patient. This interpretation is more reliable but it is only available when records are obtained from documented EP studies. For out-of-hospital records a consensus decision is the only alternative.

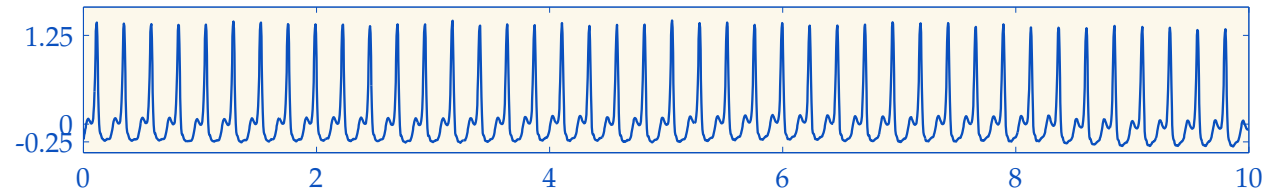

(a) Paediatric SVT with disagreements in diagnosis (Rate $255 \mathrm{bpm}$ ).

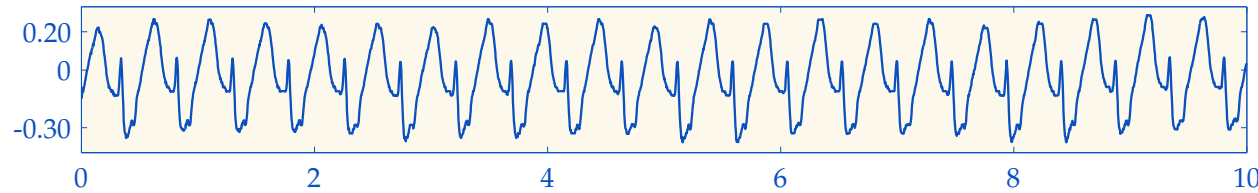

(b) Paediatric SVT with disagreements in diagnosis (Rate $125 \mathrm{bpm}$ ).

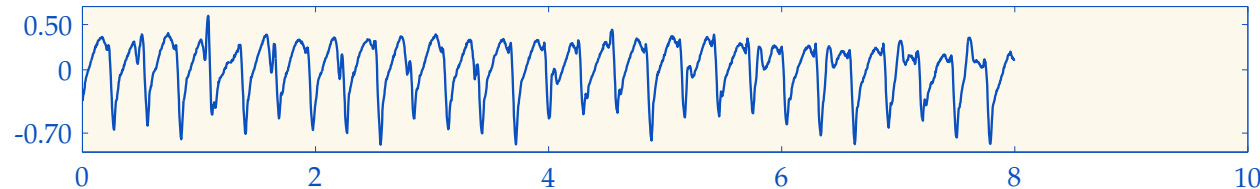

(c) Paediatric VT with disagreements in diagnosis (Rate $210 \mathrm{bpm}$ ).

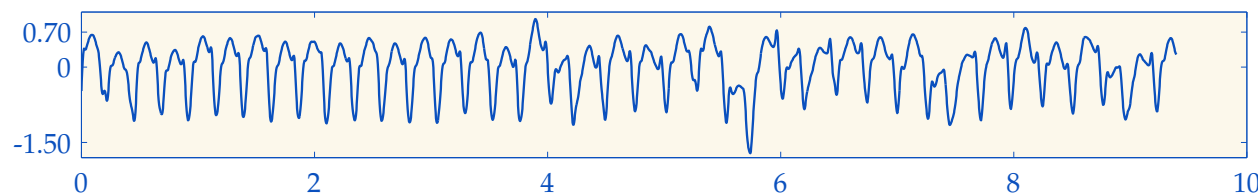

(d) Paediatric VT with disagreements in diagnosis (Rate $255 \mathrm{bpm}$ ).

Fig. 1. Examples of paediatric VT and SVT with disagreements in the cardiologists' classification. In these cases, the diagnosis from the rhythm source was adopted.

\section{Analysis of the heart rate}

A well-known ECG difference between children and adults is the heart rate. Normal heart rate changes with age, from the neonatal period, through infancy, childhood and adolescence. To maintain the cardiac output in neonates and infants, higher heart rates compensate smaller stroke volumes. As a result of the maturation of the cardiovascular system, the stroke volume increases so the heart rate lowers maintaining the overall cardiac output (Chan et al., 2008). In this section we report the heart rates of the NSR, SVT and VT records from our database. VF records were excluded from the analysis because VF is an irregular ventricular rhythm characterized by the absence of QRS complexes and a well defined heart rate. First, QRS 
complexes were automatically detected ${ }^{3}$ and the results were visually inspected and corrected when necessary. We computed the heart rate (HR) for each record as the inverse of the mean time-interval between consecutive beats (QRS complexes). The result was expressed in beats per minute (bpm).

Fig. 2 shows the HR distributions for the non-shockable (NSR and SVT) and the VT rhythms for all patients (a), the paediatric group (b) and the adult group (c). There is a remarkable overlap between the HR of the non-shockable and the VT records caused by the high rate of the non-shockable paediatric rhythms. In the adult case, the HR values of non-shockable rhythms and VT are well separated. This demonstrates that a shock/no-shock discrimination approach depending on the HR is not efficient when paediatric rhythms are included, although it can be useful for the adult group.

Table 2 presents the mean HR per rhythm category for the different age groups. As expected, HR decreases with age. Our results are in line with the literature, although the age categories do not match strictly (Chan et al., 2008; Finley \& Nugent, 1995). SVT records have a mean HR of $187 \mathrm{bpm}$, higher than the $131 \mathrm{bpm}$ obtained for the adult group. The mean HR of SVT in infants (under 1 year) is lower than the figures reported in the literature: $>220 \mathrm{bpm}$ (Chan et al., 2008) or between 260-300 bpm (Schwartz et al., 2002). For the 1-8 years of age group, the mean HR of SVT is comparable to the $>180 \mathrm{bpm}$ reported by Chan et al. The mean HR is similar for adult and paediatric VT, and is above $200 \mathrm{bpm}$ for all age groups. The overlap in HR between non-shockable and VT rhythms observed in Fig. 2 is therefore caused by the high rates of paediatric SVT. This overlap is best seen in Fig. 3 which shows the HR distributions of the paediatric VT and SVT.

\begin{tabular}{|c|c|c|c|c|c|}
\hline \multirow[b]{2}{*}{ Rhythms } & \multirow[b]{2}{*}{ Adult } & \multicolumn{4}{|c|}{ Paediatric } \\
\hline & & Total & $<1 \mathrm{y}$ & $1 y-8 y$ & $>8 y$ \\
\hline \multicolumn{6}{|c|}{ Non-shockable } \\
\hline NSR & 74 (15) & $98(21)$ & 122 (19) & 102 (19) & $90(20)$ \\
\hline SVT & $131(32)$ & $187(40)$ & $186(45)$ & 194 (39) & $180(39)$ \\
\hline \multicolumn{6}{|l|}{ Shockable } \\
\hline VT & $241(58)$ & 232 (54) & $226(31)$ & 247 (57) & $206(46)$ \\
\hline VF & - & - & - & - & - \\
\hline
\end{tabular}

Table 2. Mean HR (standard deviation in parenthesis) expressed in bpm for the adult and the paediatric records. There are no HR values for VF because it is not possible to associate a heart rate to a VF rhythm.

In conclusion, the higher heart rates of paediatric rhythms have important implications in the design of SAA. Paediatric SVT with rates close to or above those of rapid VT may be wrongly classified as shockable, therefore decreasing the specificity of the SAA.

In the next section we analyse how well several parameters discriminate shockable from non-shockable rhythms. Our purpose is not to define a SAA by optimizing the sensitivity and specificity for the records in our database. On the contrary, we study the possibility of defining

${ }^{3}$ All the signal processing and measurements for this study were made with Matlab (MathWorks, Natick, MA) 


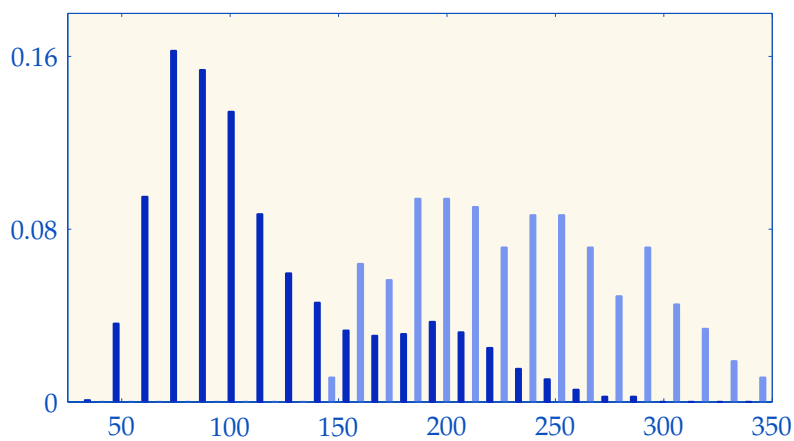

(a) HR distribution of the non-shockable ( $\mathbf{\square})$ and shockable VT

$(\square)$ records in the complete database.

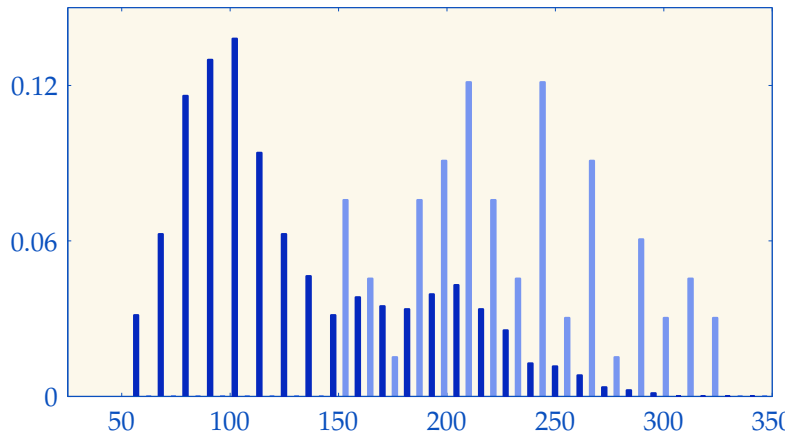

(b) HR distribution of the non-shockable ( $\square$ ) and shockable VT

( $\square$ ) records in the paediatric database.

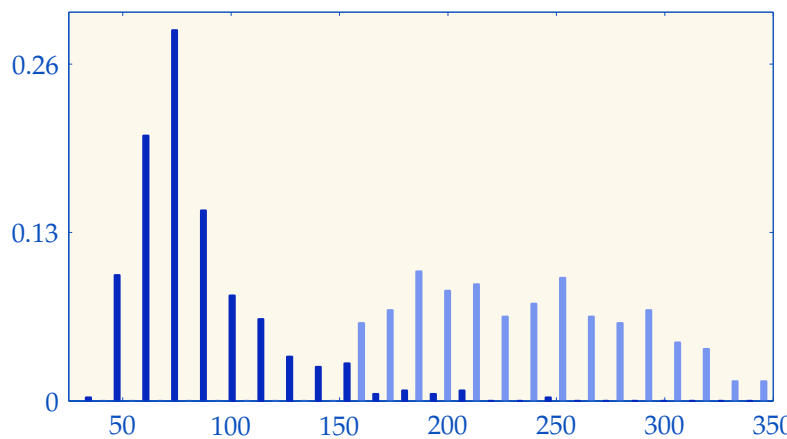

(c) HR distribution of the non-shockable ( $\square$ ) and shockable VT

(匹) records in the adult database.

Fig. 2. HR distributions for all the records, the adult records and the paediatric records. 


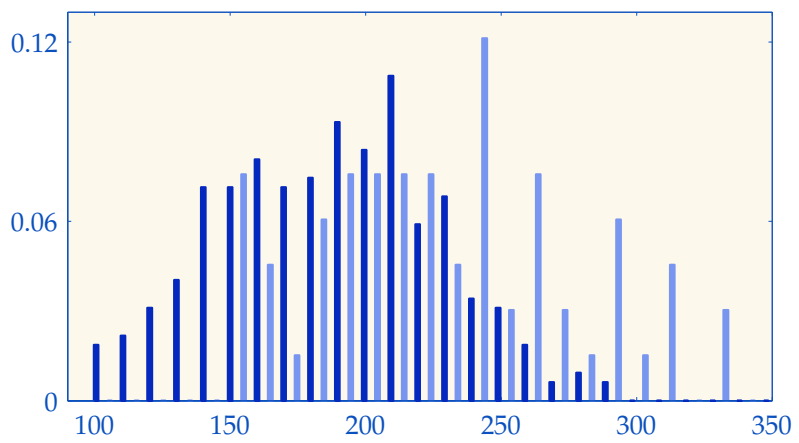

Fig. 3. HR distribution for the paediatric SVT ( $\square)$ and VT records ( $\square$ ).

different features that will serve to discriminate fatal ventricular arrhythmias regardless of the patient age, i.e., paediatric or adult. Those parameters must therefore be as independent as possible from the HR.

\section{Parameters for the shock/no-shock discrimination}

The basic principle of any SAA is the definition of a set of features that will serve to discriminate fatal ventricular arrhythmias, i.e. shockable rhythms. These features quantify distinctive characteristics of the rhythms, generally better observed by transforming the time domain representation of the ECG into a new analysis domain. For example, non-shockable rhythms have larger bandwidths and more harmonic content because of the fast-changing QRS complexes. This information is easily observed in the frequency domain representation of the ECG. Other characteristics, such as the rapid variation of the ECG waveform during QRS complexes are better observed in the slope domain.

In this section, we describe a set of four parameters and analyse their ability to accurately identify shockable arrhythmias regardless the age of the patient. One feature is computed in the slope domain; two in the frequency domain and the last one in the time domain. The parameters were computed using ECG segments of $3.2 \mathrm{~s}$ duration, and a maximum of 3 segments per record were used.

Each parameter is first analytically defined and then the idea behind the parameter is illustrated through an example from our database. Then, we compute the ROC curve for the parameter to quantify its potential ability to discriminate shockable from non-shockable rhythms. Emphasis is made on how well the parameter discriminates SVT from VT in the paediatric case. Finally, we show different graphical examples corresponding to border-line cases, for which the discrimination efficiency decreases.

\subsection{Slope domain: slope bandwidth}

In a normal sinus rhythm the ECG varies slowly most of the time, during QRS complexes however the ECG changes very rapidly. These differences in the rate of variation of the ECG permit the identification of QRS complexes in the normal ECG. In our context, we want to quantify the differences between non-shockable rhythms, with narrow QRS complexes, and shockable rhythms, with either wide QRS complexes (VT) or no QRS complexes at all (VF). 
We have defined a parameter named slope bandwidth $(s B W)$ to identify the presence of QRS complexes in the slope domain.

First, each $3.2 \mathrm{~s}$ ECG segment is preprocessed with an order 10 Butterworth band-pass filter $(0.5-30 \mathrm{~Hz})$. Then, the slope of the ECG is estimated as the first difference of the preprocessed ECG, $x_{e c g}[n]$. Since we are only interested in the relative magnitude of the slope, the first difference is squared and normalized to amplitude one.

$$
\begin{gathered}
x_{d}[n]=\left(x_{e c g}[n+1]-x_{e c g}[n]\right)^{2} \\
\overline{x_{d}}[n]=\frac{x_{d}[n]}{\max \left\{x_{d}[n]\right\}}
\end{gathered}
$$

ECG segments with QRS complexes will show large differences in the values of $\overline{x_{d}}[n]$, with peaks around the QRS complexes and valleys for the intervals when the ECG changes slowly. On the contrary, the values of $\overline{x_{d}}[n]$ are more evenly distributed for shockable rhythms because there are no narrow fast changing QRS complexes.

To quantify these differences we define the $s B W$ parameter as the $25^{\text {th }}$ percentile $\left(P_{25}\right)$ of the values of $\overline{x_{d}}[n]$ scaled by a factor of 1000 :

$$
s B W=1000 \cdot P_{25}
$$

The value of $s B W$ will be small when narrow QRS complexes are present and large when wide QRS complexes or no QRS complexes are present. Fig. 4 shows the $\overline{x_{d}}[n]$ waveforms for a non-shockable (a) and a shockable segment (b); the figure illustrates how representing the ECG in the slope domain enhances the presence of QRS complexes.

Table 3 presents the mean $s B W$ per rhythm category for the adult and paediatric records, including the 1-8 years of age group. The average $s B W$ results show a clear separation between the shockable and the non-shockable segments for each age category. As expected, $s B W$ is much larger for VT and VF segments, because shockable rhythms have more evenly distributed amplitudes in the slope domain. However, the mean $s B W$ value for paediatric VT is significantly lower than for adult VT (8.85 versus 17.99$)$. Furthermore, SVT rhythms have larger $s B W$ values, particularly in the paediatric case. Consequently, the least accurate discrimination based on the slope domain occurs between paediatric VT and SVT.

The shock/no-shock discrimination power of $s B W$ is assessed in terms of its ROC curve, shown in Fig. 5. The ROC curve depicts the proportion of correctly classified shockable segments (sensitivity) against the proportion of wrongly classified non-shockable segments (1-specificity) as the classification threshold (the value of $s B W$ ) varies. The optimal operating point (OOP) was selected to maximize the detection accuracy (number of correct decisions), which produced a $95.5 \%$ sensitivity and $97.2 \%$ specificity.

Although the global discrimination results are good a detailed analysis reveals that the SVT/VT discrimination is poorer. The SVT/VT discrimination results for the OOP obtained for the complete database were: $96.5 \%$ VT sensitivity and $94.8 \%$ SVT specificity in adults and $84.6 \%$ VT sensitivity and $92.2 \%$ SVT specificity in children.

Fig. 6 shows two examples of misclassified VT paediatric segments (the group with lowest performance). The VT segment in panel (a) presents spiky noise that can be interpreted as QRS 
complexes. The VT segment in panel (b) shows abrupt descents with large slopes, therefore is classified as non-shockable.
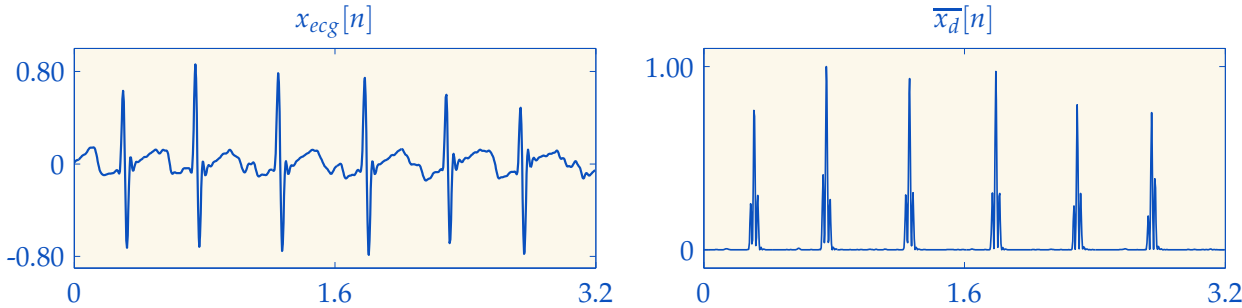

(a) SVT segment. Large differences in the slope produce low $s B W$ values $(0.06)$.
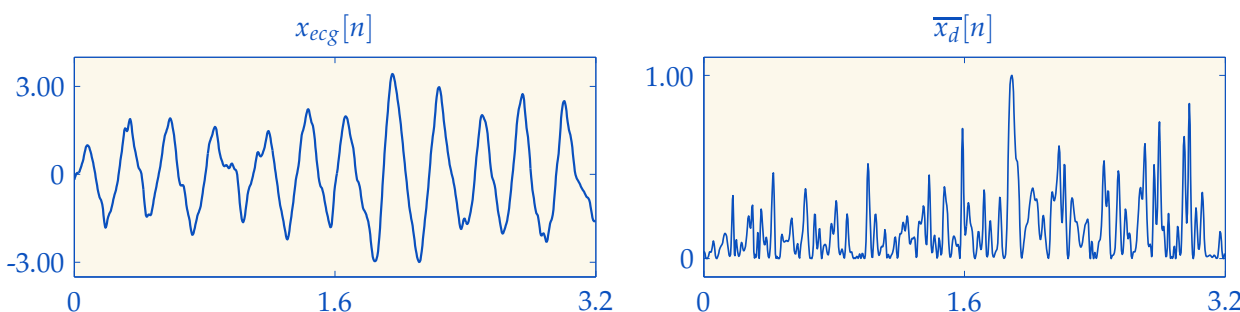

(b) VF segment. Fast ventricular activity with an evenly distributed slope produce high $s B W$ values (34.21).

Fig. 4. Differences in the distribution of the slope, $\overline{x_{d}}[n]$, between shockable and non-shockable segments.

\begin{tabular}{lcccc}
\hline & & & \multicolumn{2}{c}{ Paediatric } \\
\cline { 5 - 5 } Rhythms & All patients & Adult & Total & $1 \mathrm{y}-8 \mathrm{y}$ \\
\hline Non-shockable & & & & \\
$\quad$ NSR & $0.18(0.35)$ & $0.22(0.50)$ & $0.16(0.22)$ & $0.16(0.21)$ \\
$\quad$ SVT & $0.76(1.27)$ & $0.65(1.28)$ & $0.80(1.26)$ & $0.79(0.99)$ \\
Shockable & & & & \\
$\quad$ VT & $15.80(17.24)$ & $17.99(18.58)$ & $8.85(9.01)$ & $8.52(8.77)$ \\
VF & $10.66(7.05)$ & $10.74(7.20)$ & $10.15(6.00)$ & $9.40(4.57)$ \\
\hline
\end{tabular}

Table 3. Mean $s B W$ (standard deviation in parenthesis) for the adult and the paediatric records. 


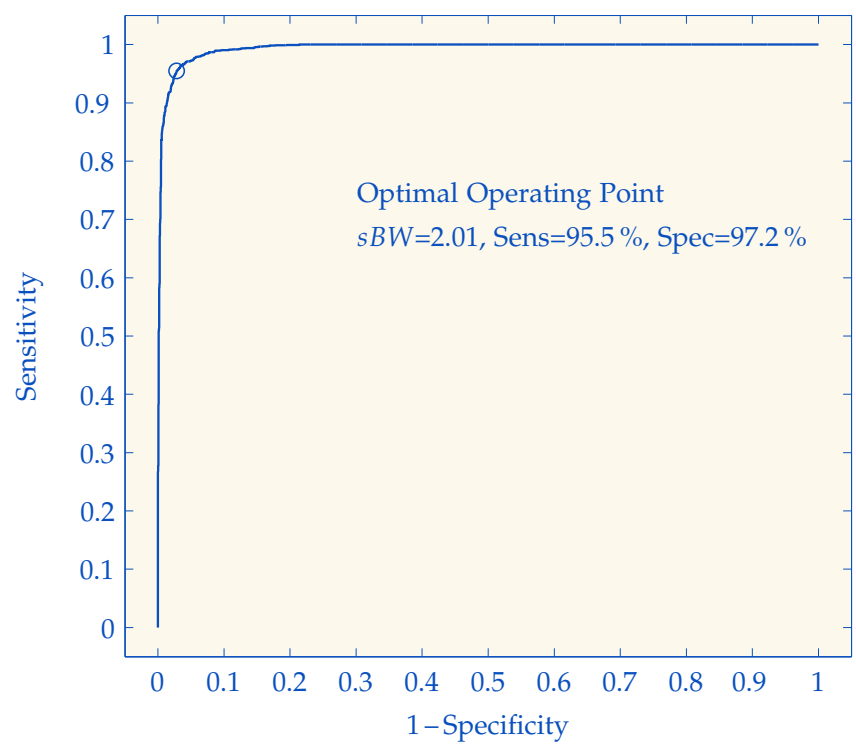

Fig. 5. ROC curve for the shock/no-shock discrimination based on the $s B W$ parameter.
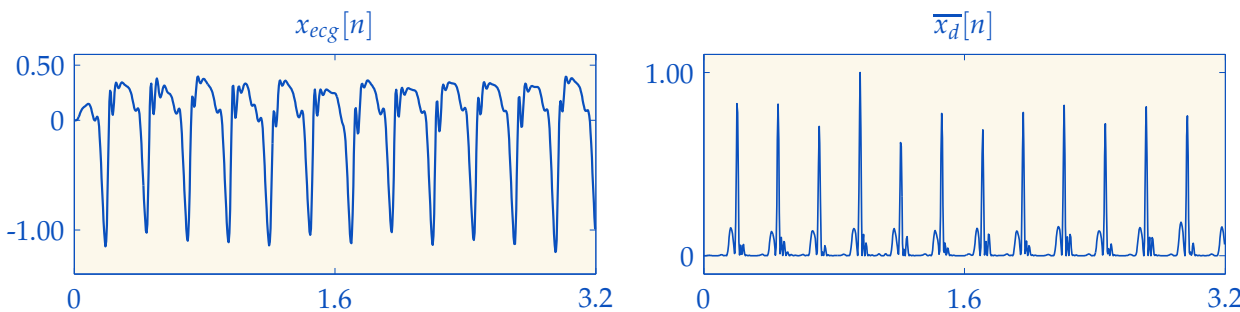

(a) VT segment with QRS like activity $(s B W=0.55)$.
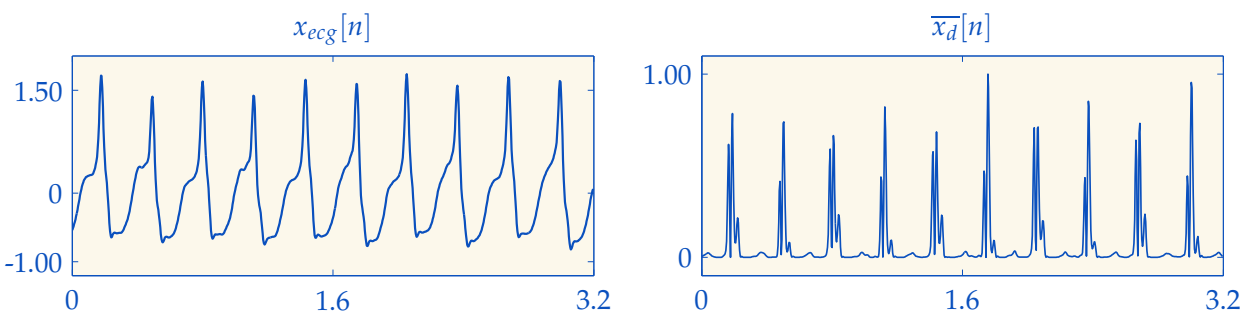

(b) VT segment with abrupt descents provoking large slopes $(s B W=0.98)$.

Fig. 6. Examples of paediatric VT segments misclassified in terms of the $s B W$ parameter. 


\subsection{Frequency domain: dominant power and high power content}

There are important differences in the power spectral density (PSD) between shockable and non-shockable rhythms. VT presents regular ventricular beats that frequently appear as a sinus-like waveform; its power spectral distribution is therefore concentrated around the frequency of the ventricular rate, the dominant frequency. VF is a more irregular rhythm so it has a wider band power distribution. Non-shockable rhythms, SVT or NSR, are very repetitive in time, and present complex waveforms consisting of a P wave, a QRS complex and $\mathrm{T}$ wave. Their power is distributed among the harmonics of the heart rate, and occupies a much larger bandwidth than for shockable rhythms. We have defined two frequency domain features to quantify these differences.

First, each $3.2 \mathrm{~s}$ ECG segment is preprocessed with an order 10 Butterworth band-pass filter $(0.5-30 \mathrm{~Hz})$. Then, we apply a Hanning window with no overlap to the segment and compute the FFT of the windowed ECG segment, $X_{e c g}(f)$. The PSD is estimated as the square of the amplitude of the FFT normalised to a unit area under the curve:

$$
\operatorname{PSD}(f)=\frac{\left|X_{e c g}(f)\right|^{2}}{\sum_{f=0}^{f=30}\left|X_{e c g}(f)\right|^{2}}
$$

The first parameter, named $P_{D}$, measures how concentrated the spectrum is around the frequency for which the PSD is maximum, the dominant frequency $f_{D}$. $P_{D}$ is the relative power content in a $B_{f}=1.2 \mathrm{~Hz}$ bandwidth symmetrically distributed around $f_{D}$ :

$$
P_{D}(\%)=100 \cdot \sum_{f_{D}-B_{f} / 2}^{f_{D}-B_{f} / 2} \operatorname{PSD}(f)
$$

The second parameter measures the power content of the high frequencies. For a cutoff frequency $f_{H}=12.5 \mathrm{~Hz}$, the relative power content above $f_{H}$ is:

$$
P_{H}(\%)=100 \cdot \sum_{f=12.5}^{f=30} \operatorname{PSD}(f)
$$

Fig. 7 shows examples of the PSD for the four type of rhythms, and the graphical description of the two parameters used to quantify these differences. VT rhythms concentrate most of the power around $f_{D}$, consequently $P_{D}$ is large and $P_{H}$ is small. For VF rhythms the power concentrates in a wider band around $f_{D}$ and the power content in the high frequencies is higher than for VT. On the contrary, rhythms with narrow QRS complexes distribute their power around several harmonics of the heart rate, $P_{D}$ is therefore smaller and $P_{H}$ larger than for shockable rhythms. The differences in the spectral separation of the harmonics between NSR and SVT rhythms are explained by the larger heart rates of SVT.

Tables 4 and 5 list the mean $P_{D}$ and $P_{H}$ for the adult and paediatric records, including the 1-8 years of age group. There is a significant difference in the values of $P_{H}$ between adult and paediatric NSR segments. This difference is due to the influence of heart rate in the power content above a fixed $f_{H}$. Children have faster normal rates, the harmonics of the heart rate are therefore at higher frequencies and the value of $P_{H}$ is larger. In any case, these values are well-above those obtained for the shockable segments. 

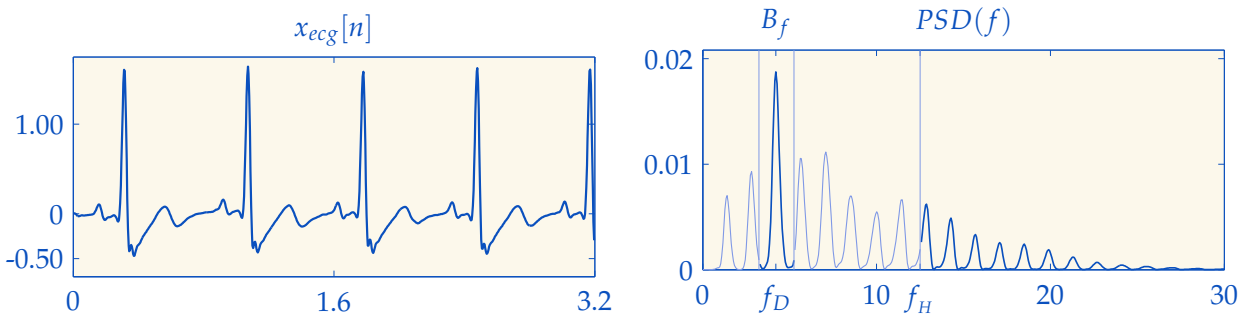

(a) NSR segment. Narrow QRS complexes produce a large harmonic content, and the power content of the high frequencies is large: $P_{D}=17.8 \%$ and $P_{H}=23.1 \%$.
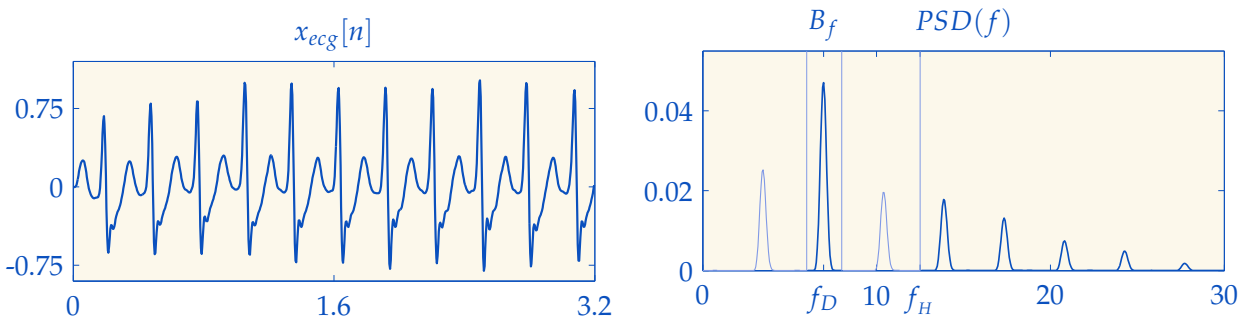

(b) SVT segment. Narrow QRS complexes produce a large harmonic content but at higher frequencies due to the larger heart rates: $P_{D}=33.5 \%$ and $P_{H}=33.8 \%$.
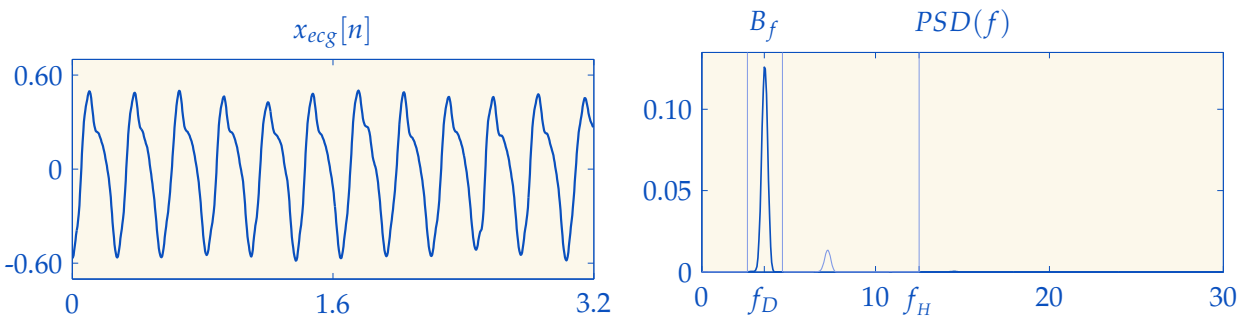

(c) VT segment. Most of the power is concentrated around $f_{D}$ and there is very little power in the high frequencies: $P_{D}=89.1 \%$ and $P_{H}=0.49 \%$.
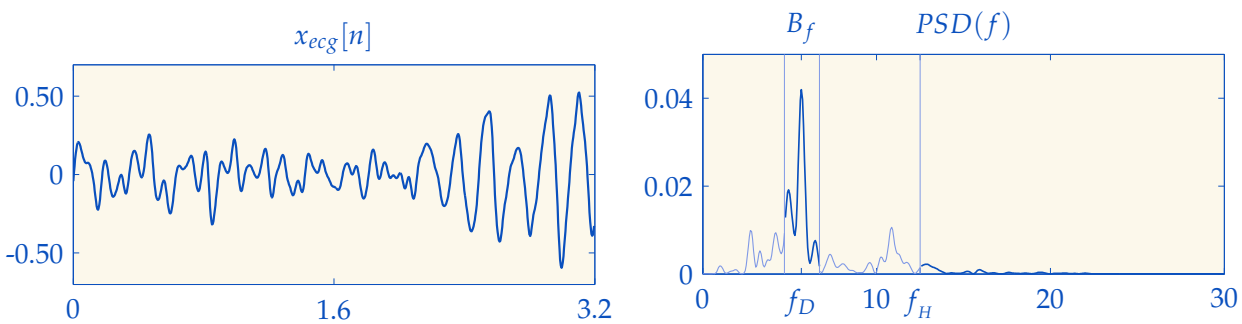

(d) VF segment. The power concentrates around $f_{D}$ but with a wider band power distribution, the power content of the high frequencies is low: $P_{D}=31.6 \%$ and $P_{H}=7.2 \%$.

Fig. 7. Examples of the PSD for the four types of rhythms. The differences in the PSD are reflected in the values of the $P_{D}$ and $P_{H}$ parameters. 


\begin{tabular}{lcccc}
\hline & & & \multicolumn{2}{c}{ Paediatric } \\
\cline { 5 - 5 } Rhythms & All patients & Adult & Total & $1 \mathrm{y}-8 \mathrm{y}$ \\
\hline Non-shockable & & & & \\
$\quad$ NSR & $19.4(8.8)$ & $20.2(9.6)$ & $19.0(8.2)$ & $18.7(8.2)$ \\
$\quad$ SVT & $32.6(14.4)$ & $28.9(14.6)$ & $33.7(14.2)$ & $35.1(13.8)$ \\
Shockable & & & & \\
$\quad$ VT & $82.4(10.2)$ & $83.5(10.1)$ & $79.0(9.8)$ & $79.5(9.1)$ \\
VF & $58.2(18.6)$ & $58.3(18.4)$ & $57.0(20.0)$ & $46.2(19.8)$ \\
\hline
\end{tabular}

Table 4. Mean $P_{D}$ (standard deviation in parenthesis) for the adult and the paediatric records.

\begin{tabular}{lcccc}
\hline & & & \multicolumn{2}{c}{ Paediatric } \\
\cline { 5 - 5 } Rhythms & All patients & Adult & Total & $1 \mathrm{y}-8 \mathrm{y}$ \\
\hline Non-shockable & & & & \\
$\quad$ NSR & $27.2(14.4)$ & $21.6(13.4)$ & $30.5(13.4)$ & $33.0(12.1)$ \\
$\quad$ SVT & $28.4(15.0)$ & $25.3(16.9)$ & $29.3(14.3)$ & $28.8(14.0)$ \\
Shockable & & & & \\
$\quad$ VT & $2.0(2.1)$ & $1.6(1.9)$ & $3.1(2.3)$ & $3.3(2.4)$ \\
VF & $2.5(3.0)$ & $2.2(2.4)$ & $4.3(5.2)$ & $4.1(3.5)$ \\
\hline
\end{tabular}

Table 5. Mean $P_{H}$ (standard deviation in parenthesis) for the adult and the paediatric records.

The shock/no-shock discrimination power of $P_{D}$ and $P_{H}$ is assessed in terms of their ROC curves, shown in Figs. 8 and 9. The sensitivity and specificity for the OOP were $83.4 \%$ and $92.4 \%$ for the $P_{D}$ parameter, and $93.4 \%$ and $89.6 \%$ for the $P_{H}$ parameter, respectively. Table 6 shows the sensitivity and the specificity in the SVT/VT discrimination for the OOP threshold, both for the adult and the paediatric groups. The $P_{D}$ parameter has a very high VT sensitivity for the paediatric $(100 \%)$ and adult $(99.0 \%)$ cases $^{4}$, at the expense of a very poor SVT specificity $(79.0 \%$ and $85.8 \%$, respectively). However, in the same conditions, the $P_{H}$ parameter presents a contrary behaviour for the paediatric $(79.6 \%$ sensitivity vs $95.6 \%$ specificity) and adult cases ( $94.9 \%$ sensitivity vs $82.4 \%$ specificity).

\begin{tabular}{cccccc}
\hline \multirow{2}{*}{ SVT/VT discrimination } & \multicolumn{2}{c}{$P_{D}$} & & \multicolumn{2}{c}{$P_{H}$} \\
\cline { 2 - 3 } \cline { 5 - 6 } \cline { 5 - 6 } & Paediatric & Adult & & Paediatric & Adult \\
\hline VT sensitivity & $100 \%$ & $99.0 \%$ & & $79.6 \%$ & $95.0 \%$ \\
SVT specificity & $79.0 \%$ & $85.8 \%$ & & $95.6 \%$ & $82.4 \%$ \\
\hline
\end{tabular}

Table 6. SVT/VT discrimination based on the spectral parameters for the adult and the paediatric cases.

\footnotetext{
${ }^{4}$ VF rhythms are therefore responsible for the total sensitivity decrease, as their values are close to the SVT values, see Table 4.
} 


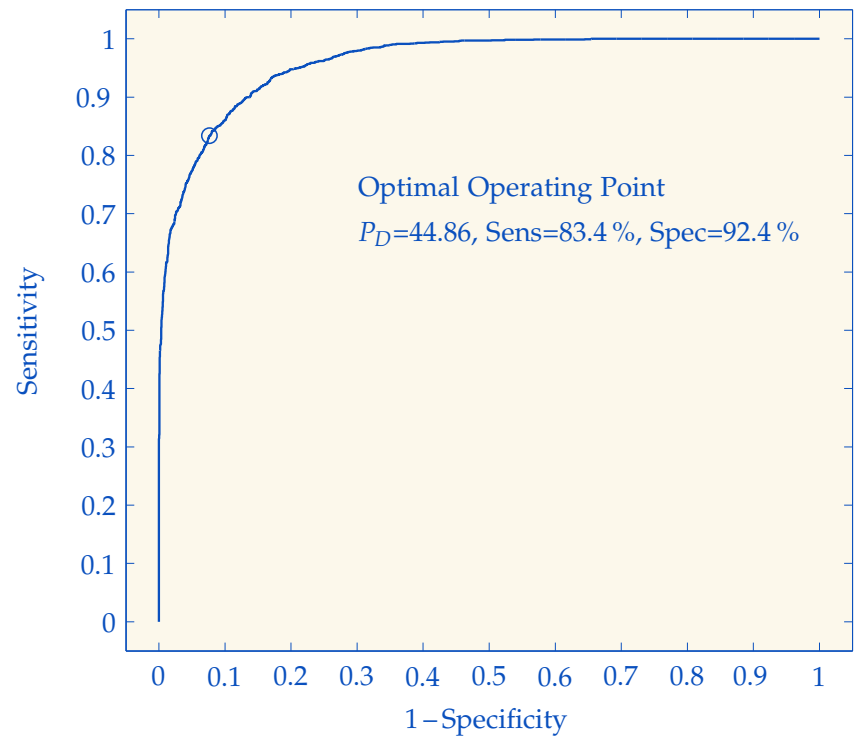

Fig. 8. ROC curve for the shock/no-shock discrimination based on the $P_{D}$ parameter.

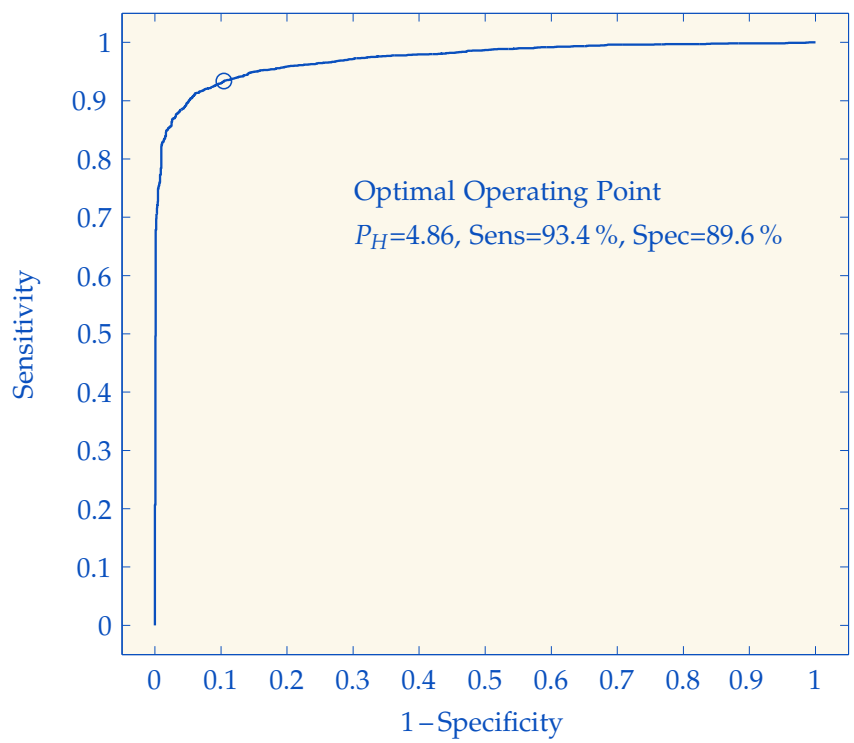

Fig. 9. ROC curve for the shock/no-shock discrimination based on the $P_{H}$ parameter. 
Fig. 10 shows one example of misclassification for each parameter in the frequency domain. The SVT segment shown in panel (a) has a large harmonic content; however, its power content around $f_{D}$ is still high $\left(P_{D}=67.7 \%\right)$. The VT segment in panel (b) has a very high heart rate (395 bpm, $6.6 \mathrm{~Hz})$, its second harmonic falls above $f_{H}$ and $P_{H}$ is therefore large.
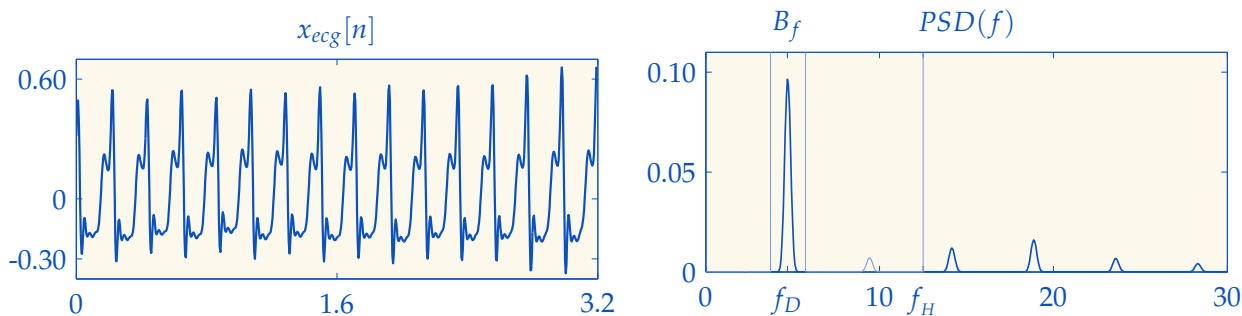

(a) SVT segment with a large power content around the fundamental frequency, $P_{D}=67.7 \%$.
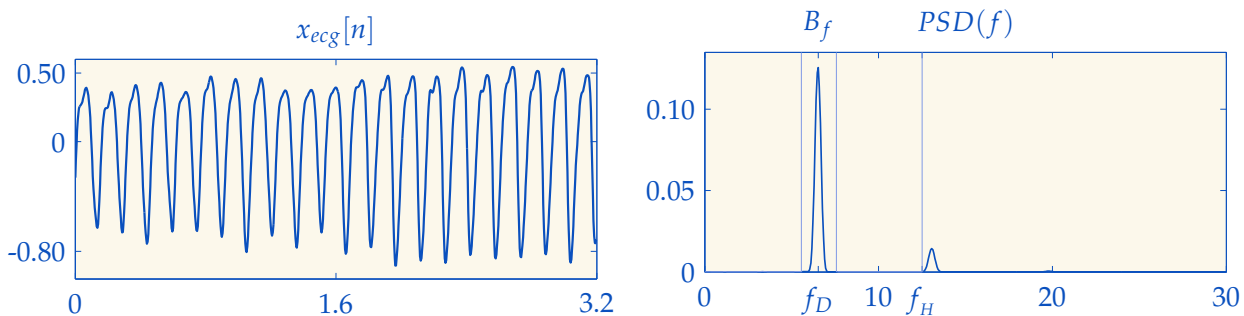

(b) VT segment. Fast rate, a significant second harmonic power content above $f_{H}, P_{H}=11.1 \%$.

Fig. 10. Examples of misclassification in the frequency domain parameters.

\subsection{Time domain: baseline content}

A normal ECG with well defined QRS complexes has long ECG intervals around the baseline or isoelectric line. These intervals shorten as the heart rate increases. For shockable rhythms, on the contrary, the proportion of time spent by the ECG around the baseline is low because of the fast ventricular activity. This difference between non-shockable and shockable rhythms is related to the presence or absence of QRS complexes. We have defined a parameter named baseline content $(b C)$. The purpose is not to accurately estimate the isoelectric content but rather to define a parameter that enhances the differences between rhythms with large and small isoelectric contents.

First, each $3.2 \mathrm{~s}$ ECG segment is preprocessed with an order 10 Butterworth band-pass filter $(5-30 \mathrm{~Hz})$. The unusually large low cutoff frequency was selected to eliminate $\mathrm{P}$ and $\mathrm{T}$ waves, highlighting the presence of QRS complexes and maximizing the baseline effect. The preprocessed ECG is then normalised so that the maximum absolute value of the amplitude of $x_{e c g}[n]$ is one,

$$
\overline{x_{e c g}}[n]=\frac{x_{e c g}[n]}{\max \left\{\left|x_{e c g}[n]\right|\right\}}
$$

The $b C$ parameter is computed in the time domain as the proportion of the samples of $\overline{x_{e c g}}[n]$ in the \pm 0.1 range. Fig. 11 shows an example of the calculation of this parameter. Segments 

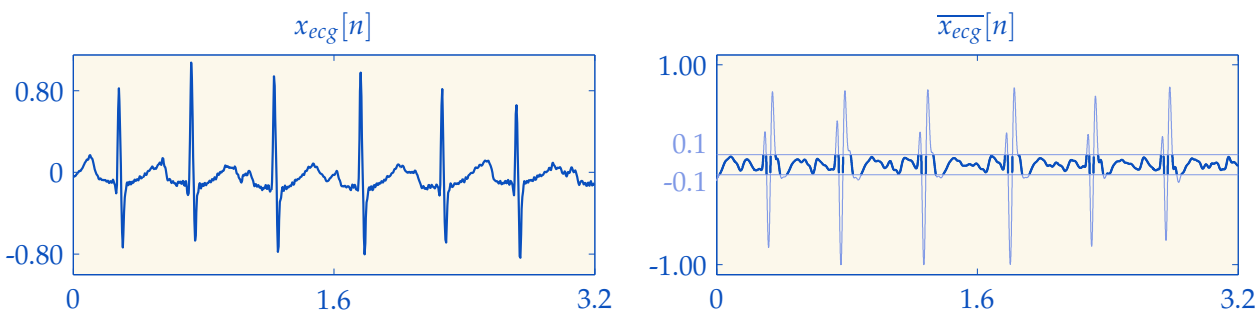

(a) SVT segment. Long isoelectric intervals between QRS complexes; $b C=0.75$
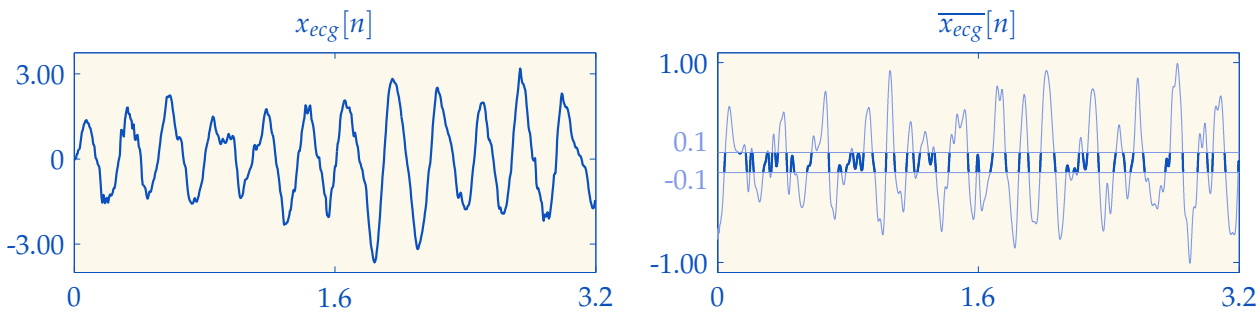

(b) VF segment. Fast ventricular activity with low isoelectric content; $b C=0.20$

Fig. 11. Differences in the value of $b C$ for shockable and non-shockable segments. The waveform of the normalised preprocessed ECG, $\overline{x_{e c g}}(n)$, is very distorted because of the large low cutoff frequency of the preprocessing filter.

with QRS complexes contain a large proportion of samples in the \pm 0.1 range, $b C$ is therefore large. On the other hand, fast ventricular arrhythmias present evenly distributed amplitudes and $b C$ is small.

Table 7 presents the mean $b C$ per rhythm category for the adult and paediatric records, including the 1-8 years of age group. For each rhythm type, the differences in the value of $b C$ between the age groups are small. The largest difference occurs between adult and paediatric SVT, and is caused by the large difference in HR reported in table 2. As in the other two domains paediatric SVT is the most difficult to discriminate rhythm.

The shock/no-shock discrimination power of $b C$ is assessed in terms of the ROC curves, shown in Fig. 12. The total sensitivity and specificity for the OOP were $91.1 \%$ and $92.0 \%$. Again the SVT/VT discrimination, particularly for paediatric patients, remains a problem. Paediatric VT sensitivity is good, $93.2 \%$, the SVT specificity however is a poor $72.2 \%$. The figures are better in the adult case, $95.5 \%$ VT sensitivity and $85.8 \%$ SVT specificity, although far from reliable for a SAA.

Fig. 13 shows two examples of misclassified SVT paediatric segments. The SVT segment in panel (a) presents large T waves, the baseline content is therefore low. The SVT segment in panel (b) shows a similar effect for a faster rate. 


\begin{tabular}{lllll}
\hline & & & \multicolumn{2}{c}{ Paediatric } \\
\cline { 5 - 5 } Rhythms & All patients & Adult & Total & $1 \mathrm{y}-8 \mathrm{y}$ \\
\hline Non-shockable & & & & \\
$\quad$ NSR & $0.65(0.13)$ & $0.67(0.14)$ & $0.64(0.13)$ & $0.65(0.12)$ \\
$\quad$ SVT & $0.40(0.14)$ & $0.49(0.16)$ & $0.37(0.13)$ & $0.35(0.11)$ \\
Shockable & & & & \\
$\quad$ VT & $0.17(0.07)$ & $0.16(0.07)$ & $0.19(0.06)$ & $0.17(0.05)$ \\
VF & $0.21(0.07)$ & $0.21(0.07)$ & $0.22(0.06)$ & $0.23(0.07)$ \\
\hline
\end{tabular}

Table 7. Mean $b C$ (standard deviation in parenthesis) for the total, the adult and the paediatric records, including the 1-8 years old subgroup.

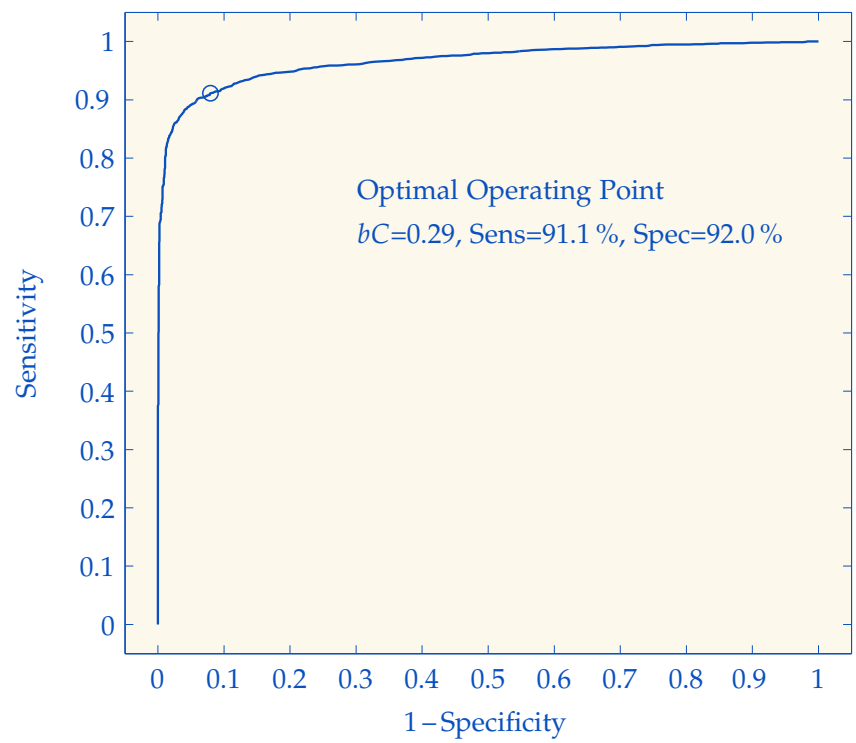

Fig. 12. ROC curve for the shock/no-shock discrimination based on the $b C$ parameter. 

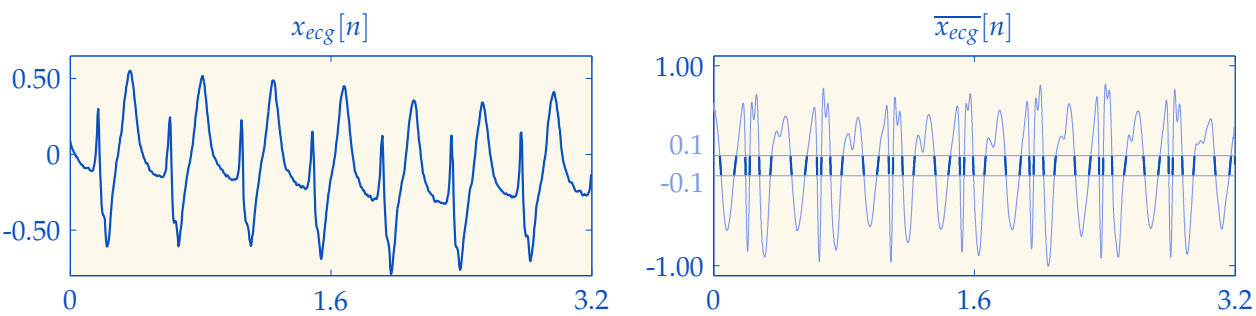

(a) SVT segment with large T waves $(b C=0.08)$.
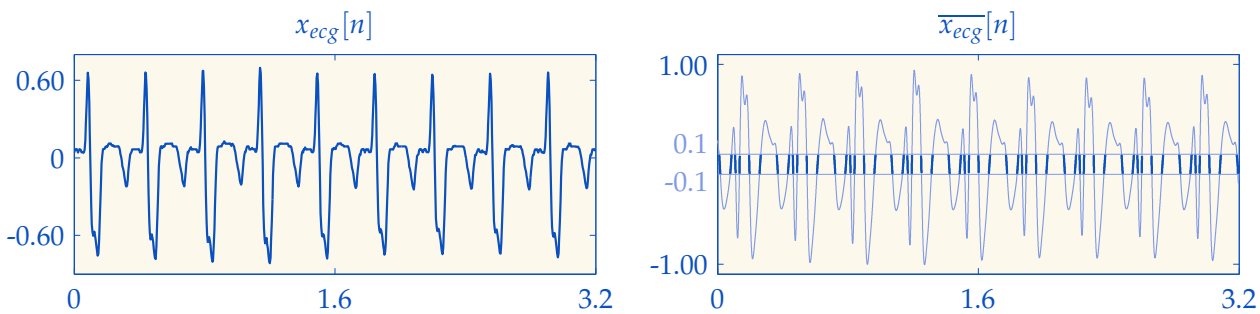

(b) SVT segment with low baseline content $(b C=0.09)$.

Fig. 13. Examples of paediatric SVT segments misclassified in terms of the $b C$ parameter.

\section{Discussion and conclusions}

The ILCOR recommended the use of AED in children 1-8 years of age in 2003. Two independent studies provided the scientific evidence for this recommendation (Atkinson et al., 2003; Cecchin et al., 2001). These contributions demonstrated that two adult algorithms from commercial AED accurately identified paediatric non-shockable rhythms and paediatric VF. In both studies, the overall specificity was above $99 \%$ and the VF sensitivity above $95 \%$. However, both studies failed in the accurate classification of rapid VT; Cecchin et al. reported a $71 \%$ VT sensitivity, and the results from Atkinson et al. were not significant because their database only contained three instances of shockable VT. These two studies represent the first alternative for the design of SAA adapted for paediatric use, which consists on proving that a SAA designed for adult patients is suitable for paediatric patients

The differences between paediatric and adult arrhythmias might explain the poor VT sensitivity results; in particular, the higher rates of paediatric SVT. A related problem is that, as indicated in the ILCOR statement, heart rate oriented SAA designed for adult patients might identify high rate paediatric SVT as shockable. Atkins et al. (Atkins et al., 2008) addressed this difficulty when they showed how an SAA designed for adult patients failed to accurately identify non-shockable paediatric SVT, the SVT specificity was $87 \%$. They increased the SVT specificity to $99.6 \%$ by applying specific detection criteria to paediatric rhythms. This study proposes a second alternative for the design of paediatric SAA, namely changing the detection criteria fixed for adult patients to accurately detect paediatric arrhythmias.

The current literature only covers ventricular arrhythmia detection algorithms developed and tested using arrhythmias from adult patients, and the validation of complete AED SAA in children. In this work, we have analysed the feasibility of a third alternative, oriented to the 
design of a SAA but including adult and paediatric rhythms in the design of the algorithm. Consequently, a significant database of adult and paediatric arrhythmias is needed from the early stages of the process. Obtaining shockable paediatric arrhythmias is particularly challenging because fatal ventricular arrhythmias are scarce in children. We describe the process of gathering and classifying a database of adult and paediatric rhythms compliant with the AHA statement. This laborious and complex task involved emergency services, hospitals and, in particular, expert cardiologists to classify the records and biomedical engineers to manage and store the data.

We have dedicated a section to analyse the heart rate of the records in our database, and to asses the differences in heart rate between children and adults. Our analysis shows that the discrimination of shockable rhythms based on the heart rate may be accurate with adult patients but not valid for children, due to the higher rates of paediatric SVT. Consequently, rate-oriented SAA designed for adults will present a low specificity for paediatric SVT, which confirms the findings of Atkins et al (Atkins et al., 2008).

The core of the chapter is dedicated to the analysis of the ECG in the slope, time and frequency domains. We define four easily computable parameters to quantify the differences between shockable and non-shockable rhythms. These parameters were designed to be independent of the heart rate and therefore less influenced by the age of the patient.

After the analysis, we conclude that the parameters are to a great degree independent of the heart rate. There are, however, small differences between paediatric and adult patients for some rhythm types. For example, the average value of the $s B W$ parameter for VT is different in adults and children. We have quantified the discrimination capacity of the four parameters in terms of their ROC curves. As anticipated, there are some borderline cases between paediatric SVT and VT. In fact, the accurate discrimination of paediatric SVT is a key aspect when adapting adult AED algorithms for paediatric use. Following this line, Irusta and Ruiz proposed a SVT/VT discrimination algorithm that could be incorporated to adult and paediatric AED (Irusta \& Ruiz, 2009).

Although the performance of each parameter is not sufficient for a reliable shock/no-shock discrimination, the parameters can be efficiently combined to accurately identify non-shockable rhythms with narrow QRS complexes. However, a SAA valid for adult and paediatric patients based on this strategy must incorporate several additional algorithms which fall beyond the scope of this work. Those algorithms include: an algorithm for the detection of non-shockable rhythms with low electrical activity such as asystole or idioventricular rhythms, an algorithm to discriminate VF (always shockable) from VT (shockable above a rate threshold) and the protective addition of a SVT/VT discrimination algorithm to avoid shock diagnoses for fast paediatric SVT.

\section{References}

Aramendi, E., Irusta, U., Pastor, E. et al. (2010). ECG spectral and morphological parameters reviewed and updated to detect adult and paediatric life-threatening arrhythmia., Physiological Measurement 31(6): 749-761.

Atkins, D. L., Scott, W. A., Blaufox, A. D. et al. (2008). Sensitivity and specificity of an automated external defibrillator algorithm designed for pediatric patients., Resuscitation 76(2): 168-174. 
Atkinson, E., Mikysa, B., Conway, J. A. et al. (2003). Specificity and sensitivity of automated external defibrillator rhythm analysis in infants and children., Ann Emerg Med 42(2): 185-196.

Biarent, D., Bingham, R., Eich, C. et al. (2010). European Resuscitation Council Guidelines for Resuscitation 2010 Section 6. Paediatric life support., Resuscitation 81(10): 1364-1388.

Biarent, D., Bingham, R., Richmond, S. et al. (2005). European Resuscitation Council guidelines for resuscitation 2005. Section 6. Paediatric life support., Resuscitation 67 Suppl 1: S97-133.

Cecchin, F., Jorgenson, D. B., Berul, C. I. et al. (2001). Is arrhythmia detection by automatic external defibrillator accurate for children?: Sensitivity and specificity of an automatic external defibrillator algorithm in 696 pediatric arrhythmias., Circulation 103(20): 2483-2488.

Chan, T. C., Sharieff, G. Q. \& Brady, W. J. (2008). Electrocardiographic manifestations: pediatric ECG., J Emerg Med 35(4): 421-430.

Finley, J. P. \& Nugent, S. T. (1995). Heart rate variability in infants, children and young adults., J Auton Nerv Syst 51(2): 103-108.

Irusta, U. \& Ruiz, J. (2009). An algorithm to discriminate supraventricular from ventricular tachycardia in automated external defibrillators valid for adult and paediatric patients., Resuscitation 80(11): 1229-1233.

Irusta, U., Ruiz, J., Aramendi, E. \& de Gauna, S. R. (2008). Amplitude, frequency and complexity features in paediatric and adult ventricular fibrillation., Resuscitation 77: S53-S53.

Jacobs, I., Nadkarni, V., Bahr, J. et al. (2004). Cardiac arrest and cardiopulmonary resuscitation outcome reports: update and simplification of the Utstein templates for resuscitation registries., Circulation 110(21): 3385-3397.

Kerber, R. E., Becker, L. B., Bourland, J. D. et al. (1997). Automatic external defibrillators for public access defibrillation: recommendations for specifying and reporting arrhythmia analysis algorithm performance, incorporating new waveforms, and enhancing safety. A statement for health professionals from the American Heart Association Task Force on Automatic External Defibrillation, Subcommittee on AED Safety and Efficacy., Circulation 95(6): 1677-1682.

Larsen, M. P., Eisenberg, M. S., Cummins, R. O. \& Hallstrom, A. P. (1993). Predicting survival from out-of-hospital cardiac arrest: a graphic model., Ann Emerg Med 22(11): 1652-1658.

Myerburg, R. J. (2001). Sudden cardiac death: exploring the limits of our knowledge., J Cardiovasc Electrophysiol 12(3): 369-381.

Rea, T. D., Eisenberg, M. S., Sinibaldi, G. \& White, R. D. (2004). Incidence of EMS-treated out-of-hospital cardiac arrest in the United States., Resuscitation 63(1): 17-24.

Ruiz de Gauna, S., Ruiz, J., Irusta, U. \& Aramendi, E. (2008). Parameters affecting shock decision in pediatric automated defibrillation., Proc. Computers in Cardiology, pp. 929-932.

Rustwick, B., Geheb, F., Brewer, J. \& Atkins, D. (2007). Electrocardiographic characteristics for automated external defibrillator algorithms are different between children and adults., Circulation 116. 
Samson, R. A., Berg, R. A., Bingham, R. et al. (2003). Use of automated external defibrillators for children: an update: an advisory statement from the pediatric advanced life support task force, International Liaison Committee on Resuscitation., Circulation 107(25): 3250-3255.

Schwartz, P. J., Garson, A., Paul, T. et al. (2002). Guidelines for the interpretation of the neonatal electrocardiogram. A task force of the European Society of Cardiology., Eur Heart J 23(17): 1329-1344.

Sirbaugh, P. E., Pepe, P. E., Shook, J. E. et al. (1999). A prospective, population-based study of the demographics, epidemiology, management, and outcome of out-of-hospital pediatric cardiopulmonary arrest., Ann Emerg Med 33(2): 174-184.

Valenzuela, T. D., Roe, D. J., Cretin, S. et al. (1997). Estimating effectiveness of cardiac arrest interventions: a logistic regression survival model., Circulation 96(10): 3308-3313.

Waalewijn, R. A., Nijpels, M. A., Tijssen, J. G. \& Koster, R. W. (2002). Prevention of deterioration of ventricular fibrillation by basic life support during out-of-hospital cardiac arrest., Resuscitation 54(1): 31-36. 


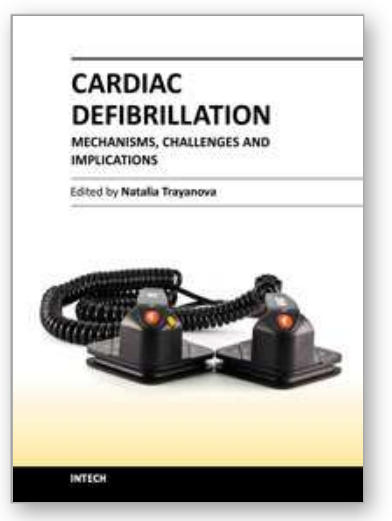

\author{
Cardiac Defibrillation - Mechanisms, Challenges and Implications \\ Edited by Prof. Natalia Trayanova
}

ISBN 978-953-307-666-9

Hard cover, 248 pages

Publisher InTech

Published online 26, September, 2011

Published in print edition September, 2011

The only known effective therapy for lethal disturbances in cardiac rhythm is deï-brillation, the delivery of a strong electric shock to the heart. This technique constitutes the most important means for prevention of sudden cardiac death. The efficacy of defibrillation has led to an exponential growth in the number of patients receiving implantable devices. The objective of this book is to present contemporary views on the basic mechanisms by which the heart responds to an electric shock, as well as on the challenges and implications of clinical defibrillation. Basic science chapters elucidate questions such as lead configurations and the reasons by which a defibrillation shock fails. Chapters devoted to the challenges in the clinical procedure of defibrillation address issues related to inappropriate and unnecessary shocks, complications associated with the implantation of cardioverter/defibrillator devices, and the application of the therapy in pediatric patients and young adults. The book also examines the implications of defibrillation therapy, such as patient risk stratification, cardiac rehabilitation, and remote monitoring of patient with implantable devices.

\title{
How to reference
}

In order to correctly reference this scholarly work, feel free to copy and paste the following:

Sofia Ruiz de Gauna, Jesus Ruiz, Unai Irusta and Elisabete Aramendi (2011). AED for Paediatric Use, Implications in the Design of Shock Advice Algorithms, Cardiac Defibrillation - Mechanisms, Challenges and Implications, Prof. Natalia Trayanova (Ed.), ISBN: 978-953-307-666-9, InTech, Available from: http://www.intechopen.com/books/cardiac-defibrillation-mechanisms-challenges-and-implications/aed-forpaediatric-use-implications-in-the-design-of-shock-advice-algorithms

\section{INTECH}

open science | open minds

\section{InTech Europe}

University Campus STeP Ri

Slavka Krautzeka 83/A

51000 Rijeka, Croatia

Phone: +385 (51) 770447

Fax: +385 (51) 686166

www.intechopen.com

\section{InTech China}

Unit 405, Office Block, Hotel Equatorial Shanghai

No.65, Yan An Road (West), Shanghai, 200040, China 中国上海市延安西路65号上海国际贵都大饭店办公楼405单元

Phone: +86-21-62489820

Fax: +86-21-62489821 
(C) 2011 The Author(s). Licensee IntechOpen. This chapter is distributed under the terms of the Creative Commons Attribution-NonCommercialShareAlike-3.0 License, which permits use, distribution and reproduction for non-commercial purposes, provided the original is properly cited and derivative works building on this content are distributed under the same license. 